\title{
Modeling, Simulation and Control of 3-DOF Redundant Fault Tolerant Robots by Means of Adaptive Inertia
}

\author{
Claudio Urrea and John Kern \\ Departamento de Ingeniería Eléctrica, DIE \\ Universidad de Santiago de Chile, USACH \\ Santiago, Chile
}

\section{Introduction}

Control systems that have the ability, in the one hand, to detect incipient failures in sensors and/ or actuators and, in the other hand, to adapt quickly the control laws in order to preserve the specified performance in terms of production quality, safety, etc., are called fault-tolerant control systems (Blanke et al., 2001), (Blanke et al., 2006). This kind of systems have become increasingly important for manipulator robots, especially those performing in remote or dangerous zones, like outer space, underwater or nuclear environments. In this context, there is a growing need and interest in developing control systems that can operate in acceptable manners, even after the occurrence of failures, also being able to stop the process before irreparable damages arise (Bonivento et al., 2004).

In this work, modeling, simulation and control of industrial fault tolerant robots by means of adaptive inertia is presented. This study, initially developed for robots with $n$ Degrees Of Freedom (DOF), includes the calculation of adaptive inertia parameters; which is particularized for planar systems with two and three rotational joints. The modeling of these systems considers kinematic and dynamic aspects of robots, including the dynamics of actuators and position sensors that, by employing MatLab/ Simulink software, permits the simulation and results displaying of dynamic behavior of such systems in front of actuator failures.

\section{Fault tolerant controller: adaptive inertia}

The active fault tolerant control system proposed in this work is based on an adaptive control law, specifically: adaptive inertia (Lewis et al., 2004), (Siciliano \& Khatib, 2008), (Ollero, 2001) then it is necessary to consider the manipulator dynamic model in the way expressed in equation (1) (Angeles, 2006), (Craig, 1986), (Spong et al., 2005), where the term corresponding to centrifugal and Coriolis forces is expressed through a matrix $\mathrm{Vm}$.

$$
\mathbf{\tau}=\mathbf{M}(q) \ddot{\mathbf{q}}+\mathbf{V}_{\mathbf{m}}(q, \dot{q}) \dot{\mathbf{q}}+\mathbf{G}(q)+\mathbf{F}(\dot{q})
$$

Position, speed and acceleration errors, in terms of manipulator link coordinates, are shown in equations (2), (3) and (4), respectively. 


$$
\begin{aligned}
& \mathbf{e}=\mathbf{q}_{\mathbf{d}}-\mathbf{q} \\
& \dot{\mathbf{e}}=\dot{\mathbf{q}}_{\mathbf{d}}-\dot{\mathbf{q}} \\
& \ddot{\mathbf{e}}=\ddot{\mathbf{q}}_{\mathbf{d}}-\ddot{\mathbf{q}}
\end{aligned}
$$

Clearing link position q, its first and second derivative; from equations (2), (3) and (4), respectively we have:

$$
\begin{aligned}
& \mathbf{q}=\mathbf{q}_{\mathbf{d}}-\mathbf{e} \\
& \dot{\mathbf{q}}=\dot{\mathbf{q}}_{\mathbf{d}}-\dot{\mathbf{e}} \\
& \ddot{\mathbf{q}}=\ddot{\mathbf{q}}_{\mathbf{d}}-\ddot{\mathbf{e}}
\end{aligned}
$$

Next, we define error auxiliar signal $\mathbf{r}$ and its derivative $\dot{\mathbf{r}}$, with respect to time, as shown in equations (8) and (9), respectively:

$$
\begin{aligned}
\mathbf{r} & =\Lambda \mathbf{e}+\dot{\mathbf{e}} \\
\dot{\mathbf{r}} & =\Lambda \dot{\mathbf{e}}+\ddot{\mathbf{e}}
\end{aligned}
$$

where:

$\Lambda$ : Definite positive diagonal matrix ( $n \times n$ dimension).

$$
\Lambda=\left[\begin{array}{llll}
\lambda_{1} & & & \\
& \lambda_{2} & & \\
& & \ddots & \\
& & & \lambda_{n}
\end{array}\right]
$$

Clearing the first and second derivatives from error in equations (8) and (9), respectively, we obtain:

$$
\begin{aligned}
& \dot{\mathbf{e}}=\mathbf{r}-\Lambda \mathbf{e} \\
& \ddot{\mathbf{e}}=\dot{\mathbf{r}}-\Lambda \dot{\mathbf{e}}
\end{aligned}
$$

Replacing equations (11) and (12) in equations (5), (6) and (7), we have:

$$
\begin{aligned}
& \dot{\mathbf{q}}=\dot{\mathbf{q}}_{\mathbf{d}}-\mathbf{r}+\Lambda \mathbf{e} \\
& \ddot{\mathbf{q}}=\ddot{\mathbf{q}}_{\mathbf{d}}-\dot{\mathbf{r}}+\Lambda \dot{\mathbf{e}}
\end{aligned}
$$

Replacing equations (13) and (14) in expression (1), we achieve: 


$$
\mathbf{\tau}=\mathbf{M}(q)\left(\ddot{\mathbf{q}}_{\mathbf{d}}-\dot{\mathbf{r}}+\Lambda \dot{\mathbf{e}}\right)+\mathbf{V}_{\mathbf{m}}(q, \dot{q})\left(\dot{\mathbf{q}}_{\mathbf{d}}-\mathbf{r}+\Lambda \mathbf{e}\right)+\mathbf{G}(q)+\mathbf{F}(\dot{q})
$$

Then:

$$
\mathbf{\tau}=\mathbf{M}(q)\left(\ddot{\mathbf{q}}_{\mathbf{d}}+\Lambda \dot{\mathbf{e}}\right)+\mathbf{V}_{\mathbf{m}}(q, \dot{q})\left(\dot{\mathbf{q}}_{\mathbf{d}}+\Lambda \mathbf{e}\right)+\mathbf{G}(q)+\mathbf{F}(\dot{q})-\mathbf{M}(q) \dot{\mathbf{r}}-\mathbf{V}_{\mathbf{m}}(q, \dot{q}) \mathbf{r}
$$

Making the following matching:

$$
\mathbf{Y}(\cdot) \boldsymbol{\varphi}=\mathbf{M}(q)\left(\ddot{\mathbf{q}}_{\mathbf{d}}+\Lambda \dot{\mathbf{e}}\right)+\mathbf{V}_{\mathbf{m}}(q, \dot{q})\left(\dot{\mathbf{q}}_{\mathbf{d}}+\Lambda \mathbf{e}\right)+\mathbf{G}(q)+\mathbf{F}(\dot{q})
$$

where:

$$
\mathbf{Y}\left(q, \dot{q}, q_{d}, \dot{q}_{d}, \ddot{q}_{d}\right)=\left[\begin{array}{cccc}
Y_{11} & Y_{12} & \cdots & Y_{1 n} \\
Y_{21} & Y_{22} & \cdots & Y_{2 n} \\
\vdots & \vdots & \ddots & \vdots \\
Y_{n 1} & Y_{n 2} & \cdots & Y_{n n}
\end{array}\right]
$$

$\mathbf{Y}(\cdot)$ : Regression matrix ( $n \times n$ dimension).

$\varphi$ : Parameter vector ( $n \times n$ dimension).

Equation (16) can be written in the following way:

$$
\mathbf{\tau}=\mathbf{Y}(\cdot) \boldsymbol{\varphi}-\mathbf{M}(q) \dot{\mathbf{r}}-\mathbf{V}_{\mathbf{m}}(q, \dot{q}) \mathbf{r}
$$

The control torque is given by:

$$
\mathbf{\tau}=\mathbf{Y}(\cdot) \hat{\boldsymbol{\varphi}}+\mathbf{K}_{\mathbf{v}} \mathbf{r}
$$

where:

$\mathrm{K}_{\mathrm{v}}$ : Definite positive diagonal matrix ( $n \times n$ dimension).

$\hat{\varphi}$ : Parameter estimation vector ( $n \times n$ dimension).

The update rule is expressed by:

$$
\dot{\hat{\boldsymbol{\varphi}}}=-\dot{\tilde{\boldsymbol{\varphi}}}=\Gamma \mathbf{Y}^{T}(\cdot) \mathbf{r}
$$

where:

$\Gamma$ : Definite positive diagonal matrix ( $n \times n$ dimension).

$$
\boldsymbol{\Gamma}=\left[\begin{array}{llll}
\gamma_{1} & & & \\
& \gamma_{2} & & \\
& & \ddots & \\
& & & \gamma_{n}
\end{array}\right]
$$

Replacing equation (21) into equation (20), we have:

$$
\mathbf{\tau}=\mathbf{Y}(\cdot) \int \Gamma \mathbf{Y}^{T}(\cdot) \mathbf{r}+\mathbf{K}_{\mathbf{v}} \mathbf{r}
$$

Replacing equation (8) into equation (23), we have:

$$
\mathbf{\tau}=\mathbf{Y}(\cdot) \int \Gamma \mathbf{Y}^{T}(\cdot)(\Lambda \mathbf{e}+\dot{\mathbf{e}})+\mathbf{K}_{\mathbf{V}} \Lambda \mathbf{e}+\mathbf{K}_{\mathbf{V}} \dot{\mathbf{e}}
$$




\section{Applications}

3.1 Planar system with two rotational joints

3.1.1 Vm matrix for 2 DOF

To obtain the matrix of centrifugal and Coriolis forces in a planar system with two rotational joints, we must consider:

$$
\begin{gathered}
\mathrm{C}(\mathbf{q}, \dot{\mathbf{q}})=\mathrm{V}_{\mathrm{m}}(\mathbf{q}, \dot{\mathbf{q}}) \dot{\mathbf{q}} \\
\mathbf{V}_{\mathrm{m}}=\frac{\mathbf{1}}{\mathbf{2}}\left(\dot{\mathbf{M}}+\mathbf{U}^{\mathrm{T}}-\mathbf{U}\right) \\
\mathbf{U}=\left(\mathbf{I} \otimes \dot{\mathbf{q}}^{T}\right) \frac{\partial \mathbf{M}}{\partial \mathbf{q}}
\end{gathered}
$$

where:

$\otimes:$ Kronecker product.

Considering the expression given by (64) (Appendix A), and developing equations (26) and (27), we have:

$$
\begin{aligned}
& \mathbf{V}_{\mathbf{m}}=\left[\begin{array}{ll}
V_{m 11} & V_{m 12} \\
V_{m 21} & V_{m 22}
\end{array}\right] \\
& \left(\mathbf{I} \otimes \dot{\mathbf{q}}^{\mathrm{T}}\right)=\left[\begin{array}{ll}
1 & 0 \\
0 & 1
\end{array}\right] \otimes\left[\begin{array}{ll}
\dot{q}_{1} & \dot{q}_{2}
\end{array}\right] \\
& \left(\mathbf{I} \otimes \dot{\mathbf{q}}^{T}\right)=\left[\begin{array}{cccc}
\dot{q}_{1} & \dot{q}_{2} & 0 & 0 \\
0 & 0 & \dot{q}_{1} & \dot{q}_{2}
\end{array}\right] \\
& \frac{\partial \mathbf{M}}{\partial \mathbf{q}}=\left[\begin{array}{l}
\frac{\partial \mathbf{M}}{\partial q_{1}} \\
\frac{\partial \mathbf{M}}{\partial q_{2}}
\end{array}\right] \\
& V_{m 11}=-m_{2} l_{1} l_{\mathrm{c} 2} \sin \theta_{2} \dot{\theta}_{2} \\
& V_{m 12}=-m_{2} l_{1} l_{\mathrm{c} 2} \sin \theta_{2}\left(\dot{\theta}_{1}+\dot{\theta}_{2}\right) \\
& V_{m 21}=m_{2} l_{1} l_{\mathrm{c} 2} \sin \theta_{2} \dot{\theta}_{1} \\
& V_{m 22}=0
\end{aligned}
$$

\subsubsection{Regression matrix for 2 DOF}

The regression matrix considering two degrees of freedom is expressed by:

$$
\mathbf{Y}=\left[\begin{array}{ll}
Y_{11} & Y_{12} \\
Y_{21} & Y_{22}
\end{array}\right]
$$


Calculation of regression matrix is developed in Appendix B, resulting in the components we can see in equation (34):

$$
\begin{aligned}
& Y_{11}=\left(\frac{I_{1 \mathrm{zz}}}{m_{1}}+l_{\mathrm{c} 1}^{2}\right) \dot{Q}_{1} \\
& Y_{12}=l_{1}^{2} \dot{Q}_{1}+\left(\frac{I_{2 \mathrm{zz}}}{m_{2}}+l_{\mathrm{c} 2}^{2}\right)\left(\dot{Q}_{1}+\dot{Q}_{2}\right)+y_{A}\left(2 \dot{Q}_{1}+\dot{Q}_{2}\right)-y_{a}\left(Q_{2}\left(\dot{\theta}_{1}+\dot{\theta}_{2}\right)+Q_{1} \dot{\theta}_{2}\right) \\
& Y_{21}=0 \\
& Y_{22}=y_{a} \dot{\theta}_{1} Q_{1}+y_{A} \dot{Q}_{1}+\left(\frac{I_{2 \mathrm{zz}}}{m_{2}}+l_{\mathrm{c} 2}^{2}\right)\left(\dot{Q}_{1}+\dot{Q}_{2}\right)
\end{aligned}
$$

\subsection{Planar system with three rotational joints}

We will consider now the three first DOF of the robotic manipulator shown in figure 1.

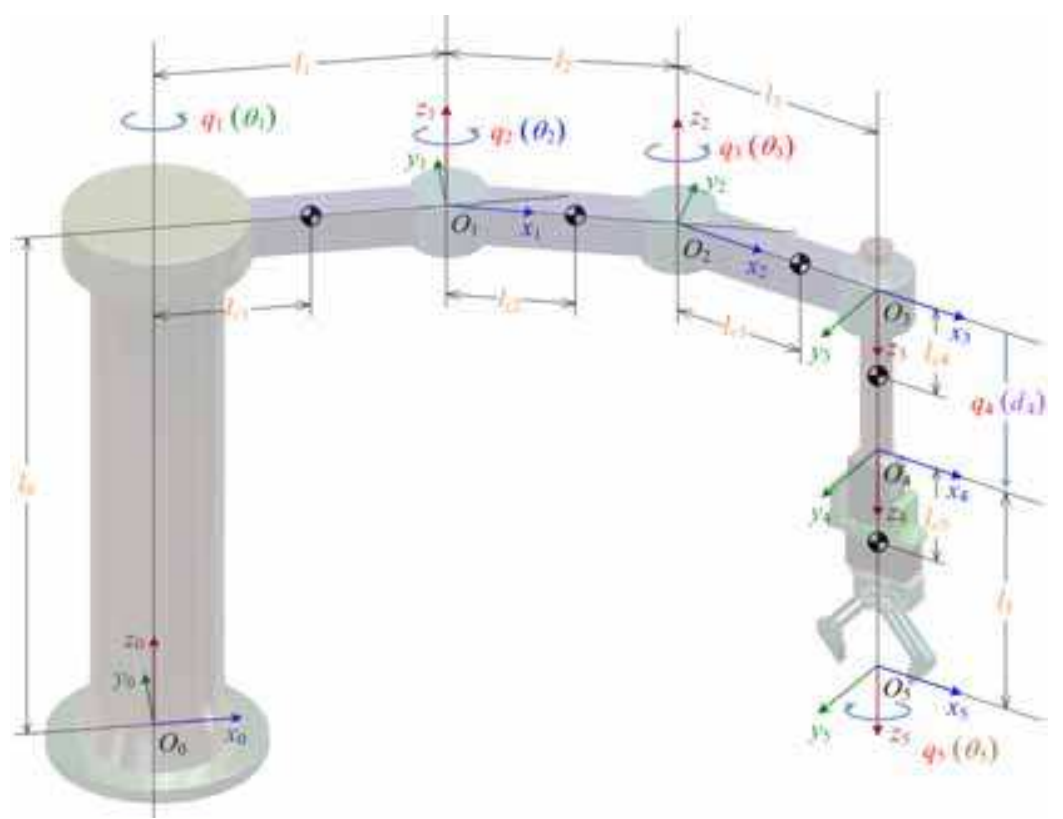

Fig. 1. Scheme of a redundant robotic manipulator of SCARA type

\subsubsection{Vm matrix for 3 DOF3}

From the development of expressions given by (26) and (27), considering three degrees of freedom, we get:

$$
\mathbf{V}_{\mathbf{m}}=\left[\begin{array}{lll}
V_{m 11} & V_{m 12} & V_{m 13} \\
V_{m 21} & V_{m 22} & V_{m 23} \\
V_{m 31} & V_{m 32} & V_{m 33}
\end{array}\right]
$$




$$
\begin{gathered}
\left(\mathbf{I} \otimes \dot{\mathbf{q}}^{T}\right)=\left[\begin{array}{ccc}
1 & 0 & 0 \\
0 & 1 & 0 \\
0 & 0 & 1
\end{array}\right] \otimes\left[\begin{array}{llllll}
\dot{q}_{1} & \dot{q}_{2} & \dot{q}_{3}
\end{array}\right] \\
\left(\mathbf{I} \otimes \dot{\mathbf{q}}^{T}\right)=\left[\begin{array}{ccccccccc}
\dot{q}_{1} & \dot{q}_{2} & \dot{q}_{3} & 0 & 0 & 0 & 0 & 0 & 0 \\
0 & 0 & 0 & \dot{q}_{1} & \dot{q}_{2} & \dot{q}_{3} & 0 & 0 & 0 \\
0 & 0 & 0 & 0 & 0 & 0 & \dot{q}_{1} & \dot{q}_{2} & \dot{q}_{3}
\end{array}\right] \\
\frac{\partial \mathbf{M}}{\partial \mathbf{q}}=\left[\begin{array}{l}
\frac{\partial \mathbf{M}}{\partial q_{1}} \\
\frac{\partial \mathbf{M}}{\partial q_{2}} \\
\frac{\partial \mathbf{M}}{\partial q_{3}}
\end{array}\right]
\end{gathered}
$$

The matrix of centrifugal and Coriolis forces for the three first degrees of freedom of the robot manipulator under study, can be expressed by means of equation (39) (see Appendix C):

$$
\begin{aligned}
V_{m 11} & =-\left(c_{a}+c_{b}\right) \dot{\theta}_{2}-c_{c} \dot{\theta}_{3}-c_{d}\left(\dot{\theta}_{2}+\dot{\theta}_{3}\right) \\
V_{m 12} & =-\left(c_{a}+c_{b}\right)\left(\dot{\theta}_{1}+\dot{\theta}_{2}\right)-c_{c} \dot{\theta}_{3}-c_{d}\left(\dot{\theta}_{1}+\dot{\theta}_{2}+\dot{\theta}_{3}\right) \\
V_{m 13} & =-\left(c_{c}+c_{d}\right)\left(\dot{\theta}_{1}+\dot{\theta}_{2}+\dot{\theta}_{3}\right) \\
V_{m 21} & =\left(c_{a}+c_{b}+c_{d}\right) \dot{\theta}_{1}-c_{c} \dot{\theta}_{3} \\
V_{m 22} & =-c_{c} \dot{\theta}_{3} \\
V_{m 23} & =-c_{c}\left(\dot{\theta}_{1}+\dot{\theta}_{2}+\dot{\theta}_{3}\right) \\
V_{m 31} & =c_{c}\left(\dot{\theta}_{1}+\dot{\theta}_{2}\right)+c_{d} \dot{\theta}_{1} \\
V_{m 32} & =c_{c}\left(\dot{\theta}_{1}+\dot{\theta}_{2}\right) \\
V_{m 33} & =0
\end{aligned}
$$

\subsubsection{Regression matrix for 3 DOF}

After obtaining the matrix of centrifugal and Coriolis forces $\mathrm{Vm}$ for the three first degrees of freedom under consideration, we proceed to calculate the corresponding regression matrix, whose form is expressed by means of equation (40):

$$
\mathbf{Y}=\left[\begin{array}{lll}
Y_{11} & Y_{12} & Y_{13} \\
Y_{21} & Y_{22} & Y_{23} \\
Y_{31} & Y_{32} & Y_{33}
\end{array}\right]
$$

The components of the regression matrix are developed in Appendix D, and the results can be expressed by:

$$
Y_{11}=\left(I_{1 \mathrm{zz}} / m_{1}+l_{\mathrm{c} 1}^{2}\right) \dot{Q}_{1}
$$




$$
\begin{gathered}
Y_{12}=-y_{a}\left(\dot{\theta}_{2} Q_{1}+\left(\dot{\theta}_{1}+\dot{\theta}_{2}\right) Q_{2}\right)+\left(I_{2 \mathrm{zz}} / m_{2}+l_{1}^{2}+l_{\mathrm{c} 2}^{2}+2 y_{A}\right) \dot{Q}_{1}+\left(I_{2 \mathrm{zz}} / m_{2}+l_{\mathrm{c} 2}^{2}+y_{A}\right) \dot{Q}_{2} \\
Y_{13}=-\left(y_{b}+y_{d}\right)\left(\dot{\theta}_{2} Q_{1}+\left(\dot{\theta}_{1}+\dot{\theta}_{2}\right) Q_{2}\right)-\left(y_{c}+y_{d}\right)\left(\dot{\theta}_{3}\left(Q_{1}+Q_{2}\right)+\left(\dot{\theta}_{1}+\dot{\theta}_{2}+\dot{\theta}_{3}\right) Q_{3}\right)+ \\
\left(I_{3 \mathrm{zz}} / m_{3}+l_{1}^{2}+l_{2}^{2}+l_{\mathrm{c} 3}^{2}+2\left(y_{B}+y_{C}+y_{D}\right)\right) \dot{Q}_{1}+\left(I_{3 \mathrm{zz}} / m_{3}+l_{2}^{2}+l_{\mathrm{c} 3}^{2}+y_{B}+2 y_{C}+y_{D}\right) \\
\dot{Q}_{2}+\left(l_{\mathrm{c} 3}^{2}+y_{C}+y_{D}\right) \dot{Q}_{3} \\
Y_{21}=0 \\
Y_{23}=\left(\begin{array}{c}
\left.y_{b}+y_{d}\right) \dot{\theta}_{1} Q_{1}-y_{c}\left(\dot{\theta}_{3}\left(Q_{2}+Q_{1}+Q_{3}\right)+\left(\dot{\theta}_{1}+\dot{\theta}_{2}\right) Q_{3}\right)+\left(I_{3 \mathrm{zz}} / m_{3}+l_{2}^{2}+l_{\mathrm{c} 3}^{2}+y_{B}+\right. \\
\left.2 y_{C}+y_{D}\right) \dot{Q}_{1}+\left(I_{3 \mathrm{zz}} / m_{3}+l_{2}^{2}+l_{\mathrm{c} 3}^{2}+2 y_{C}\right) \dot{Q}_{2}+\left(I_{3 \mathrm{zz}} / m_{3}+l_{\mathrm{c} 3}^{2}+y_{C}\right) \dot{Q}_{3}
\end{array}\right. \\
Y_{31}=0 \\
\left.\left.Y_{32}^{2}+y_{A}\right)\right) \dot{Q}_{1}+\left(I_{2 \mathrm{zz}} / m_{2}+l_{\mathrm{c} 2}^{2}\right) \dot{Q}_{2} \\
Y_{33}= \\
y_{d} \dot{\theta}_{1} Q_{1}+y_{c}\left(\dot{\theta}_{1}+\dot{\theta}_{2}\right)\left(Q_{1}+Q_{2}\right)+\left(I_{3 \mathrm{zz}} / m_{3}+l_{\mathrm{c} 33}^{2}+y_{C}+y_{D}\right) \dot{Q}_{1}+\left(I_{3 \mathrm{zz}} / m_{3}+l_{\mathrm{c} 3}^{2}+\right. \\
\left.y_{C}\right) \dot{Q}_{2}+\left(I_{3 \mathrm{zz}} / m_{3}+l_{\mathrm{c} 3}^{2}\right) \dot{Q}_{3} \quad
\end{gathered}
$$

\subsubsection{Inverse kinematics}

When a failure arises in the robot manipulator, the fault tolerant controller must reconfigure itself, carrying out real-time calculation of the new inverse kinematics that is generated. In the case of adaptive inertia control, it is necessary to calculate, in addition to the new joint positions, the new joint speeds and accelerations.

The new position of joint three is given by equation (50):

$$
\theta_{N 3}=\arccos \left(\frac{\mathbf{x}^{2}+y^{2}-l_{v}^{2}-l_{3}^{2}}{2 l_{v} l_{3}}\right)-\theta_{\gamma}
$$

The new joint speed and acceleration is given by equations (55) and (56), respectively.

$$
\begin{gathered}
u=\frac{x^{2}+y^{2}-l_{v}^{2}-l_{3}^{2}}{2 l_{v} l_{3}} \\
\dot{u}=\frac{1}{l_{v} l_{3}}(x \dot{x}+y \dot{y}) \\
\ddot{u}=\frac{1}{l_{v} l_{3}}\left(\dot{x}^{2}+\dot{y}^{2}+x \ddot{x}+y \ddot{y}\right)
\end{gathered}
$$




$$
\begin{gathered}
\theta_{N 3}=\arccos (u)-\theta_{\gamma} \\
\dot{\theta}_{N 3}=-\frac{\dot{u}}{\sqrt{1-u^{2}}} \\
\ddot{\theta}_{N 3}=-\frac{\ddot{u}}{\sqrt{1-u^{2}}}-\frac{u \dot{u}^{2}}{\left(1-u^{2}\right)^{3 / 2}}
\end{gathered}
$$

The new position of joint one is expressed by equation (57):

$$
\theta_{N 1}=\arctan \left(\frac{y}{x}\right)-\arctan \left(\frac{l_{3} \sin \left(\theta_{N 3}+\theta_{\gamma}\right)}{l_{v}+l_{3} \cos \left(\theta_{N 3}+\theta_{\gamma}\right)}\right)-\theta_{\beta}
$$

The new joint speed and acceleration is given by equations (62) and (63), respectively.

$$
\begin{gathered}
v=\frac{l_{3} \sin \left(\theta_{N 3}+\theta_{\gamma}\right)}{l_{v}+l_{3} \cos \left(\theta_{N 3}+\theta_{\gamma}\right)} \\
\dot{v}=\frac{l_{3}}{l_{v}+l_{3} \cos \left(\theta_{N 3}+\theta_{\gamma}\right)}\left(\frac{l_{3} \sin \left(\theta_{N 3}+\theta_{\gamma}\right)^{2}}{l_{v}+l_{3} \cos \left(\theta_{N 3}+\theta_{\gamma}\right)}+\frac{\cos \left(\theta_{N 3}+\theta_{\gamma}\right)}{l_{v}+l_{3} \cos \left(\theta_{N 3}+\theta_{\gamma}\right)}\right) \dot{\theta}_{N 3} \\
\ddot{v}=\frac{l_{3} \sin \left(\theta_{N 3}+\theta_{\gamma}\right)}{\left(l_{v}+l_{3} \cos \left(\theta_{N 3}+\theta_{\gamma}\right)\right)^{2}}\left(\frac{2 l_{3}^{2} \sin \left(\theta_{N 3}+\theta_{\gamma}\right)^{2}}{l_{v}+l_{3} \cos \left(\theta_{N 3}+\theta_{\gamma}\right)}+3 l_{3} \cos \left(\theta_{N 3}+\theta_{\gamma}\right)-1\right) \dot{\theta}_{N 3}^{2}+ \\
\frac{l_{3}}{l_{v}+l_{3} \cos \left(\theta_{N 3}+\theta_{\gamma}\right)}\left(\frac{l_{3} \sin \left(\theta_{\mathrm{N} 3}+\theta_{\gamma}\right)^{2}}{l_{v}+l_{3} \cos \left(\theta_{N 3}+\theta_{\gamma}\right)}+\cos \left(\theta_{N 3}+\theta_{\gamma}\right) \ddot{\theta}_{N 3}\right. \\
\theta_{N 1}=\arctan \left(\frac{y}{x}\right)-\arctan (v)-\theta_{\beta} \\
\ddot{\theta}_{N 1}=\frac{\dot{\theta}_{N 1}=\frac{x \dot{y}-\dot{x} y}{x^{2}+y^{2}}-\frac{\dot{v}}{1+v^{2}}}{\left(1+v^{2}\right)^{2}}-\frac{(x \ddot{y}-\ddot{x} y)}{\left(1+v^{2}\right)}+\frac{2\left(x y\left(\dot{x}^{2}-\dot{y}^{2}\right)-\dot{x} \dot{y}\left(x^{2}-y^{2}\right)\right)}{\left(x^{2}+y^{2}\right)} \\
\left(x^{2}+y^{2}\right)^{2}
\end{gathered}
$$

\subsubsection{Simulation curves}

To obtain the different simulation curves for the proposed control system, by employing MatLab/ Simulink software, we use the simulation diagrams presented in figure 2 and figure 3. The resulting curves are shown next: 


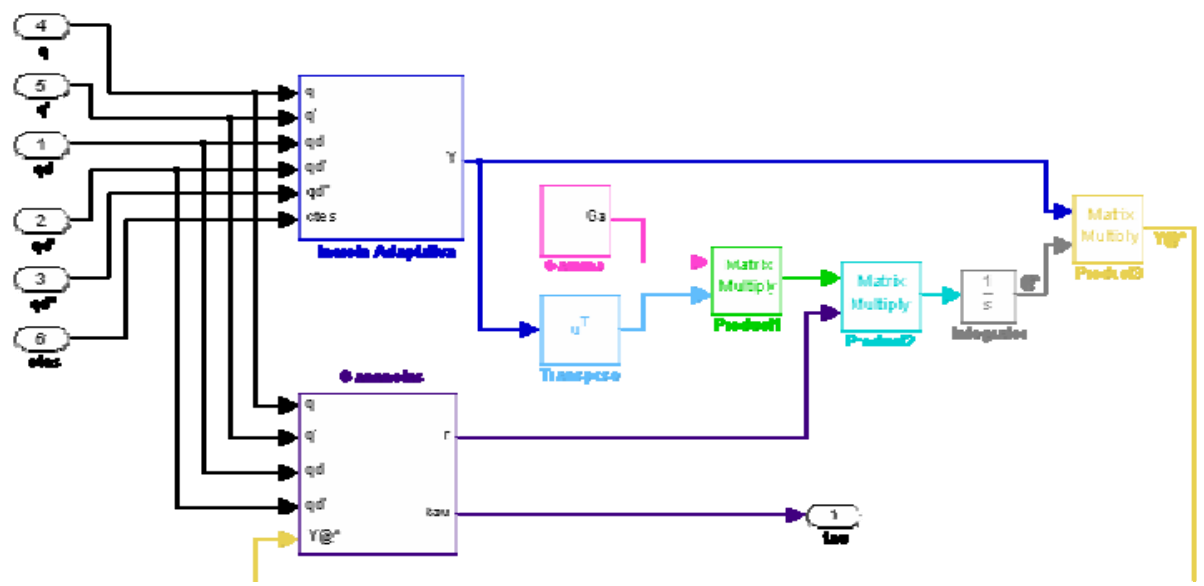

Fig. 2. Schematic diagram showing the block: adaptive inertia

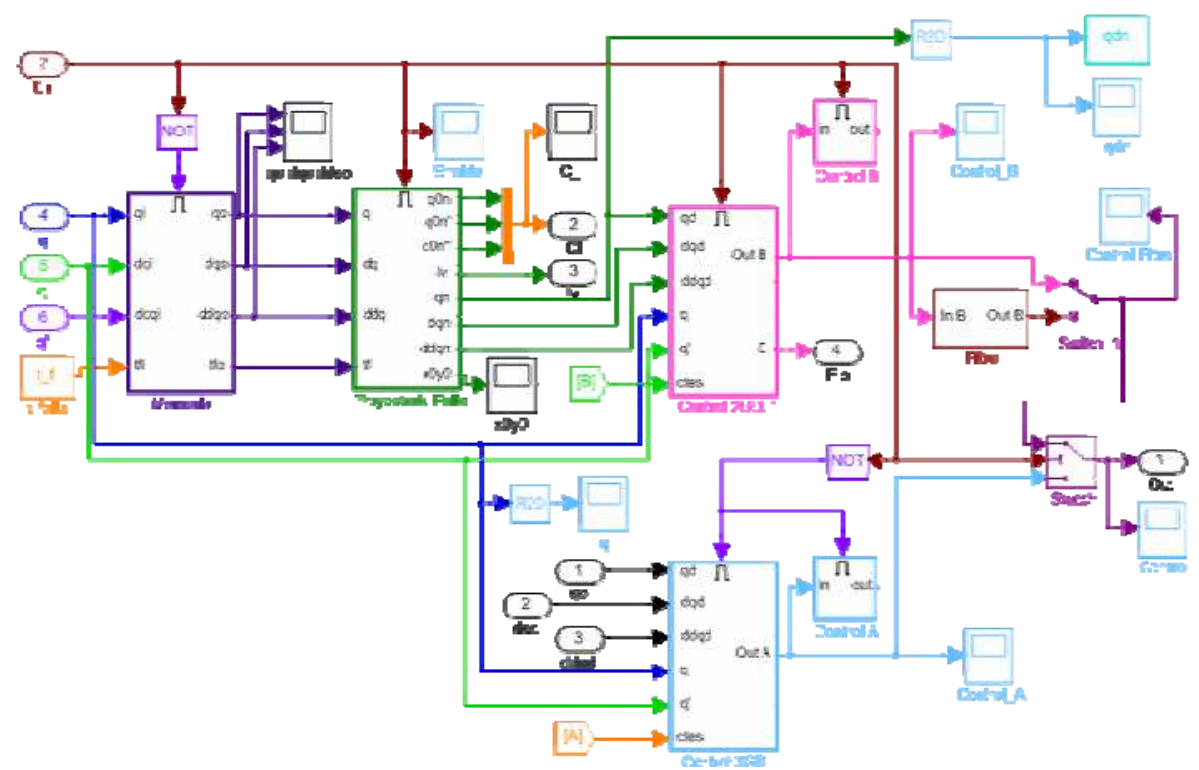

Fig. 3. Schematic diagram showing fault tolerant control stage: adaptive inertia

\subsubsection{Results}

After establishing the control laws by adaptive inertia to build fault tolerant control, we proceed to test such controller by means of the developed simulation tools. The obtained results are as follows:

Figures 4 and 5 show the test desired joint trajectory, along with the real obtained trajectory; and the test desired Cartesian trajectory along with the real obtained Cartesian trajectory. In absence of failures, we can see a good tracking of the desired trajectory. 


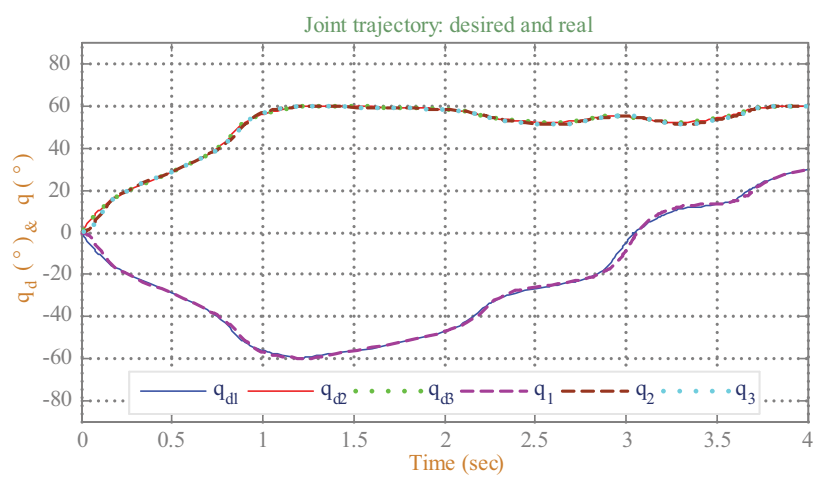

Fig. 4. Joint trajectory: desired and real

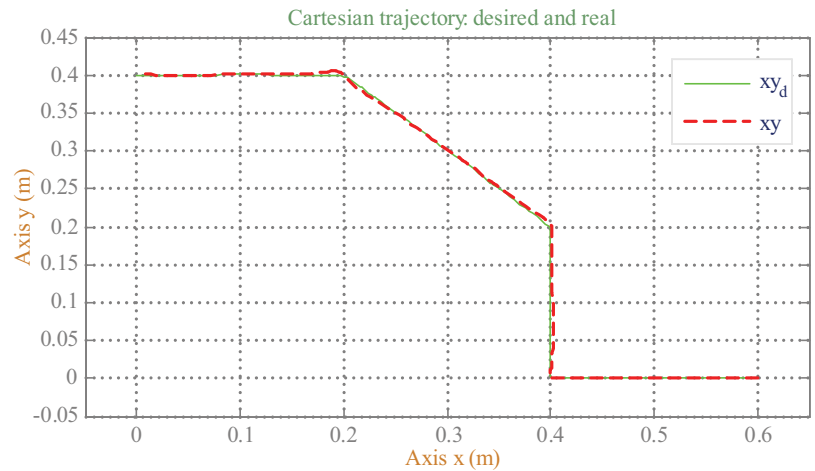

Fig. 5. Cartesian trajectory: desired and real

In figure 6 we can see the torques applied on each manipulator joint.

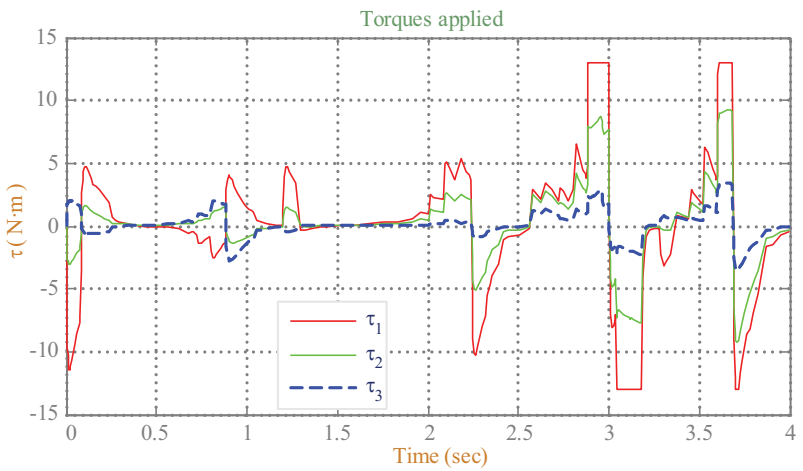

Fig. 6. Torques applied

We can notice that the greater applied torque, corresponding to joint 1 , experiences saturation due to limitations inherent to the actuator. 


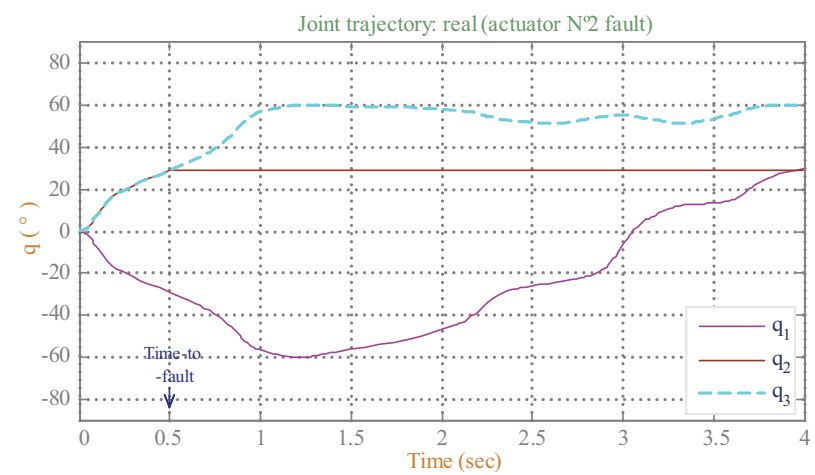

Fig. 7. Joint trajectory: real (failure in actuator № 2)

Figure 7 shows manipulator real joint trajectory when actuator 2 is blocked at 0.5 sec from starting trajectory, and figure 8 shows a superposition of real and desired joint trajectories.

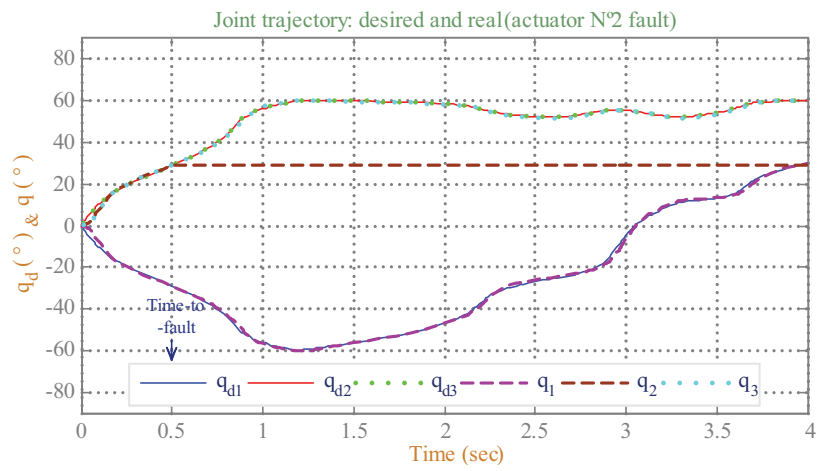

Fig. 8. Joint trajectory: desired and real (failure in actuator № 2)

The real Cartesian trajectory suffers a remarkable deviation when failure arises in actuator 2 of the manipulator, as evidenced in figure 9 .

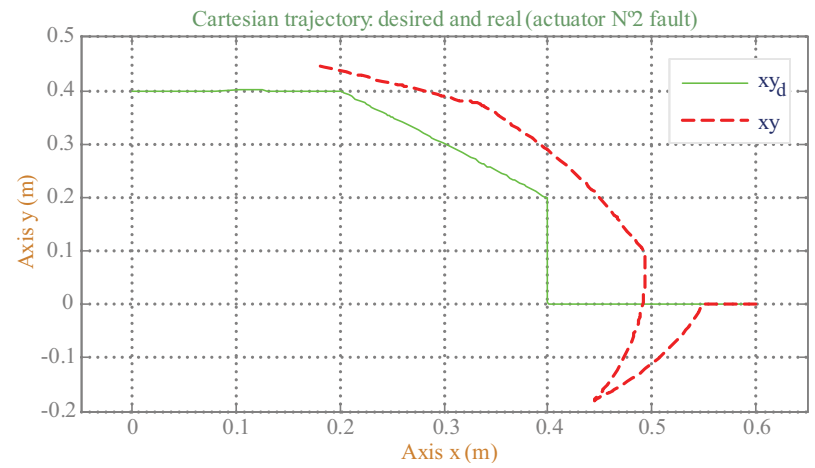

Fig. 9. Cartesian trajectory: desired and real (failure in actuator № 2 ) 
In figure 10 we can see torques applied to the robot when the controller has not corrected the fault yet (classic adaptive inertia controller: without fault tolerance).

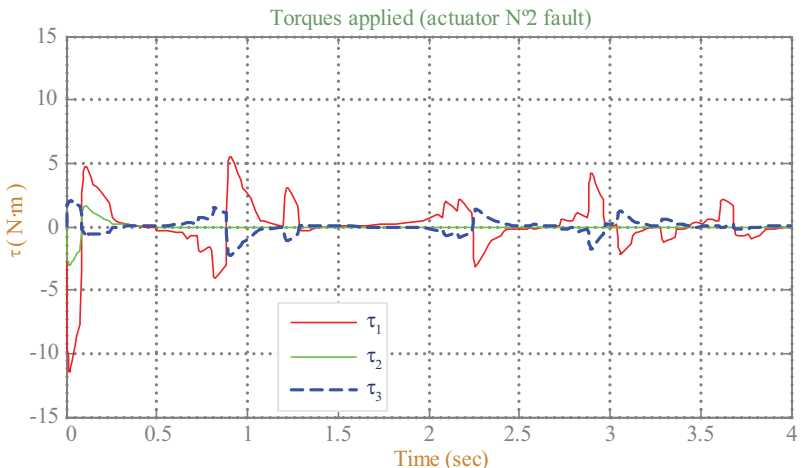

Fig. 10. Torques applied (failure in actuator № 2)

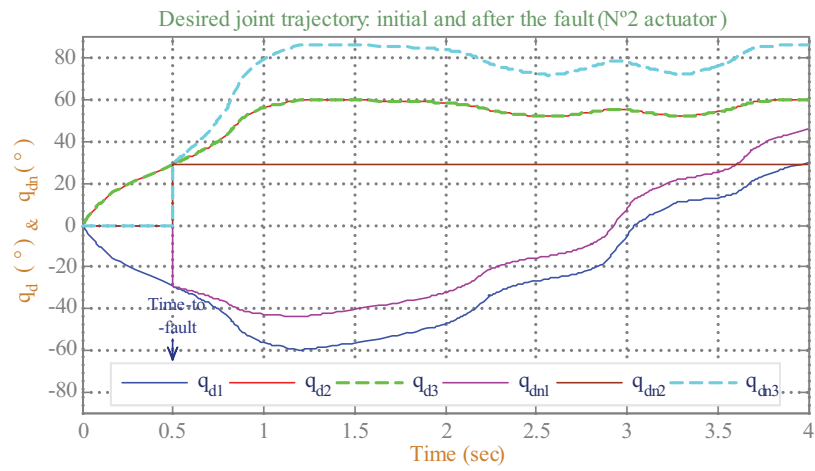

Fig. 11. Joint trajectory: desired, initial and after to failure (actuator № 2 )

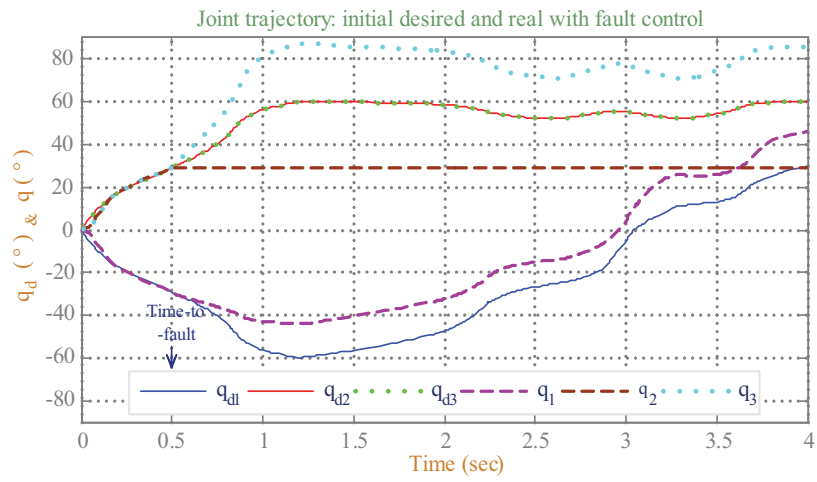

Fig. 12. Joint trajectory: desired, initial and real with fault control 


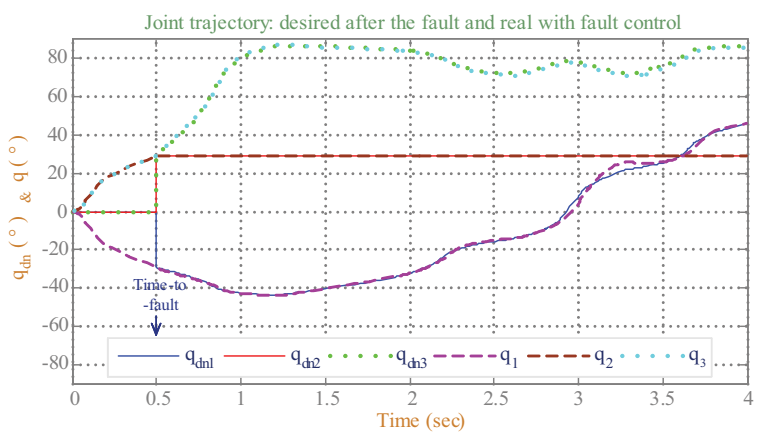

Fig. 13. Joint trajectory: desired, after the fault and real with fault control

In figure 11 we can see desired joint trajectories: initial and after to failure; in the last case, we can notice the increase in torques corresponding to joints 1 and 3 , in order to compensate the failure in actuator 2.

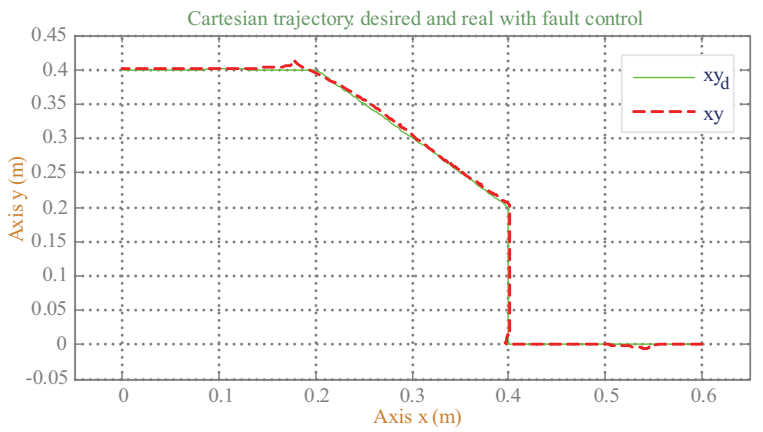

Fig. 14. Cartesian trajectory: desired and real with fault control

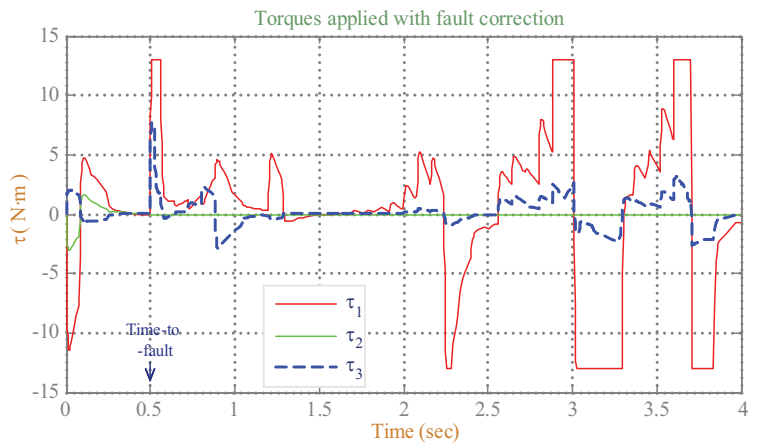

Fig. 15. Torques applied with fault correction

Figures 12 and 13 show desired, initial and real joint trajectories; and desired, after failure and real, respectively, with the application of fault tolerant control by employing adaptive inertia. 
The effectiveness of fault tolerant control by adaptive inertia is evidenced in figure 14, where we can see the desired joint trajectory along with the real joint trajectory. We can notice a remarkable improvement in the desired trajectory tracking, in comparison with the performance under adaptive inertia classic control.

In figure 15 we notice an increase in needed torques applied to joints 1 and 3 , in order to follow the desired trajectory in presence of a failure in actuator 2.

The obtained simulation curves show the better performance of the fault tolerant adaptive inertia controller when a failure arises (figure 14), in comparison with the classic adaptive inertia controller (figure 9). We can notice the influence of actuators as non-infinite torque generators in trajectory following when significant changes in joint position and speed are produced.

\section{Conclusions}

In this work we exposed the modeling and development of a fault tolerant controller by adaptive inertia, and the simulation of an industrial-type 3 DOF robot, including the application of a failure in an actuator. We presented the adaptive inertia parameter calculation, considering the manipulator kinematic and dynamic aspects. From the obtained results, we concluded that in absence of failures the classic adaptive inertia controller has a good tracking of the desired trajectory, as seen in figures 4 and 5 . The greater torque applied to the manipulator, corresponding to joint 1 , experiences saturation due to limitations inherent to the actuator, provided for the inclusion of its dynamics in the model. Classic adaptive inertia control, in presence of a failure (blocking) in actuator 2, experiences a detriment in its performance; in other words, the real Cartesian trajectory suffers a notorious deviation when a failure has been applied to the manipulator; this situation is evident in fig. 8 , and in this context the torques applied to the robot decrease significantly, as shown in fig. 10. Fault tolerant control by adaptive inertia is effective in the occurrence of a fault: the real joint trajectory approaches to the desired joint trajectory, what is evident in figure 14 . In order to compensate the immobility of failure in actuator 2 it is necessary an increase in torques corresponding to joints 1 and 3 (figure 15). We can notice the influence of actuators as generators of non-infinite torques in trajectory tracking when significant changes in joint position/ speed occur. Concluding, fault tolerant control by adaptive inertia has a better performance in desired trajectory tracking, when compared with classic adaptive inertia control.

\section{Acknowledgements}

This work had the support of the Department for Scientific and Technologic Research of the Universidad de Santiago de Chile, by means of Project 060713UO, Santiago, Chile.

\section{Further developments}

Thanks to the development of this work, from the implemented simulation tools and the obtained results, fault tolerant control systems essays are being currently carried out, in order to apply them to actual robotic systems, with and without link redundancy, like the SCARA-type robots shown in figure 16 and figure 17, respectively. 


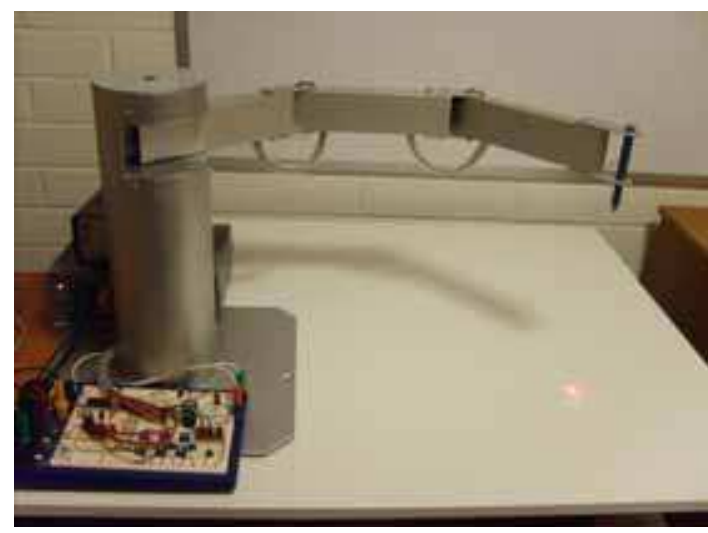

Fig. 16. SCARA-type redundant robot, DIE-USACH

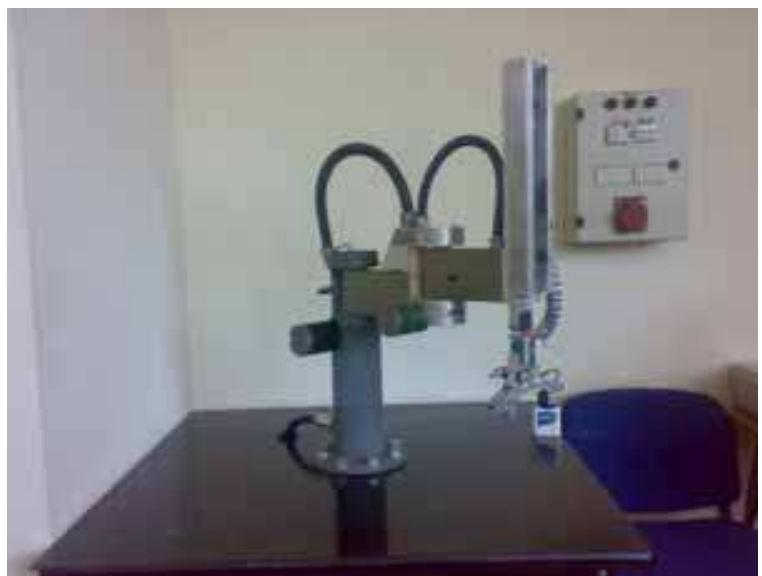

Fig. 17. SCARA-type robot, DIE-USACH

\section{References}

Angeles, J., 2006. Fundamentals of Robotic Mechanical System: Theory, Methods, and Algorithms, Third Edition. New York: Springer.

Blanke, M., Kinnaert, M., Lunze, J. \& Staroswiecki, M., 2006. Diagnosis and Fault-Tolerant Control 2nd Edition. New York: Springer-Verlag Berlin Heidelberg.

Blanke, M., Staroswiecki, M. \& Wu, E., 2001. Concepts and Methods in Fault-Tolerant Control. In Arlington, VA, USA: American Control Conference. Proceedings of the 2001., 2001. Pearson Education.

Bonivento, C., Isidori, A., Marconi, L. \& Paoli, A., 2004. Implicit Fault-Tolerant Control: Application to Induction Motors. Automatica, vol. 40, $N^{\circ} 3$, p.355-371.

Craig, J., 1986. Introduction to Robotics, Mechanics and Control. Reading, Massachusetts: Addison-Wesley Publishing Company, Inc. 
Lewis, F., Dawson, D. \& Abdallah, C., 2004. Robot Manipulator Control Theory and Practice. New York: Marcel Dekker, Inc.

Ollero, A., 2001. Robótica Manipuladores y Robots Móviles. Barcelona, España: Marcombo, S.A. Siciliano, B. \& Khatib, O., 2008. Handbook of Robotics. Berlin, Heidelberg: Springer-Verlag. Spong, M., Hutchinson, S. \& Vidyasagar, M., 2005. Robot Modeling and Control, First Edition. New York: John Wiley \& Sons, Inc.

\section{Appendices}

\subsection{Appendix A: inertia matrix for 2 DOF}

The inertia matrix considering two degrees of freedom is given by equation (64):

$$
\begin{aligned}
\mathbf{M} & =\left[\begin{array}{ll}
M_{11} & M_{12} \\
M_{21} & M_{22}
\end{array}\right] \\
M_{11} & =I_{1 \mathrm{zz}}+I_{2 \mathrm{zz}}+m_{1} l_{\mathrm{c} 1}^{2}+m_{2}\left(l_{1}^{2}+l_{\mathrm{c} 2}^{2}+2 l_{1} l_{\mathrm{c} 2} \cos \theta_{2}\right) \\
M_{12} & =I_{2 \mathrm{zz}}+m_{2}\left(l_{\mathrm{c} 2}^{2}+l_{1} l_{\mathrm{c} 2} \cos \theta_{2}\right) \\
M_{21} & =I_{2 \mathrm{zz}}+m_{2}\left(l_{\mathrm{c} 2}^{2}+l_{1} l_{\mathrm{c} 2} \cos \theta_{2}\right) \\
M_{22} & =I_{2 \mathrm{zz}}+m_{2} l_{\mathrm{c} 2}^{2}
\end{aligned}
$$

\subsection{Appendix B: regression matrix for 2 DOF}

The regression matrix considering two degrees of freedom, is expressed by equation (33). Defining relationships (65) to (68) as:

$$
\begin{aligned}
& Q_{1}=\dot{q}_{d 1}+\lambda_{1} e_{1} \\
& Q_{2}=\dot{q}_{d 2}+\lambda_{2} e_{2} \\
& \dot{Q}_{1}=\ddot{q}_{d 1}+\lambda_{1} \dot{e}_{1} \\
& \dot{Q}_{2}=\ddot{q}_{d 2}+\lambda_{2} \dot{e}_{2}
\end{aligned}
$$

The components of the matrix given by equation (33) can be calculated by means of expressions (69) to (70):

$$
\begin{aligned}
& Y_{11}=\frac{M_{11} \dot{Q}_{1}+M_{12} \dot{Q}_{2}}{m_{1}} /_{m_{2}=0}+\frac{V_{m 11} Q_{1}+V_{m 12} Q_{2}}{m_{1}} /_{m_{2}=0}+\frac{G_{1}}{m_{1}} /_{m_{2}=0} \\
& Y_{12}=\frac{M_{11} \dot{Q}_{1}+M_{12} \dot{Q}_{2}}{m_{2}} /_{m_{1}=0}+\frac{V_{m 11} Q_{1}+V_{m 12} Q_{2}}{m_{2}} /_{m_{1}=0}+\frac{G_{1}}{m_{2}} /_{m_{1}=0} \\
& Y_{21}=\frac{M_{21} \dot{Q}_{1}+M_{22} \dot{Q}_{2}}{m_{1}} /_{m_{2}=0}+\frac{V_{m 21} Q_{1}+V_{m 22} Q_{2}}{m_{1}} /_{m_{2}=0}+\frac{G_{2}}{m_{1}} /_{m_{2}=0}
\end{aligned}
$$




$$
Y_{22}=\frac{M_{21} \dot{Q}_{1}+M_{22} \dot{Q}_{2}}{m_{2}} /_{m_{1}=0}+\frac{V_{m 21} Q_{1}+V_{m 22} Q_{2}}{m_{2}} /_{m_{1}=0}+\frac{G_{2}}{m_{2}} /_{m_{1}=0}
$$

where:

$$
\mathbf{G}=\left[\begin{array}{l}
0 \\
0
\end{array}\right]
$$

With these considerations, the resulting regression matrix components are:

$$
\begin{gathered}
Y_{11}=\left(\frac{I_{1 \mathrm{zz}}}{m_{1}}+l_{\mathrm{c} 1}^{2}\right) \dot{Q}_{1} \\
Y_{12}=l_{1}^{2} \dot{Q}_{1}+\left(\frac{I_{2 \mathrm{zz}}}{m_{2}}+l_{\mathrm{c} 2}^{2}\right)\left(\dot{Q}_{1}+\dot{Q}_{2}\right)+l_{1} l_{\mathrm{c} 2} \cos \theta_{2}\left(2 \dot{Q}_{1}+\dot{Q}_{2}\right)-l_{1} l_{\mathrm{c} 2} \sin \theta_{2}\left(Q_{2}\left(\dot{\theta}_{1}+\dot{\theta}_{2}\right)+Q_{1} \dot{\theta}_{2}\right) \\
Y_{21}=0 \\
Y_{22}=l_{1} l_{\mathrm{c} 2} \sin \theta_{2} \dot{\theta}_{1} Q_{1}+l_{1} l_{\mathrm{c} 2} \cos \theta_{2} \dot{Q}_{1}+\left(\frac{I_{2 \mathrm{zz}}}{m_{2}}+l_{\mathrm{c} 2}^{2}\right)\left(\dot{Q}_{1}+\dot{Q}_{2}\right)
\end{gathered}
$$

Defining relationships (78) and (79) as:

$$
\begin{aligned}
& y_{a}=l_{1} l_{\mathrm{c} 2} \sin \theta_{2} \\
& y_{A}=l_{1} l_{\mathrm{c} 2} \cos \theta_{2}
\end{aligned}
$$

The components $Y_{12}$ and $Y_{22}$ of the regression matrix can be expressed by:

$$
\begin{gathered}
Y_{12}=l_{1}^{2} \dot{Q}_{1}+\left(\frac{I_{2 \mathrm{zz}}}{m_{2}}+l_{\mathrm{c} 2}^{2}\right)\left(\dot{Q}_{1}+\dot{Q}_{2}\right)+y_{A}\left(2 \dot{Q}_{1}+\dot{Q}_{2}\right)-y_{a}\left(Q_{1} \dot{\theta}_{2}+Q_{2}\left(\dot{\theta}_{1}+\dot{\theta}_{2}\right)\right) \\
Y_{22}=y_{a} \dot{\theta}_{1} Q_{1}+y_{A} \dot{Q}_{1}+\left(\frac{I_{2 \mathrm{zz}}}{m_{2}}+l_{\mathrm{c} 2}^{2}\right)\left(\dot{Q}_{1}+\dot{Q}_{2}\right)
\end{gathered}
$$

\subsection{Appendix C: Vm matrix for 3 DOF}

The Vm Matrix for $3 \mathrm{DOF}$ is expressed by:

$$
\begin{gathered}
V_{m 11}=-\left(m_{2} l_{1} l_{\mathrm{c} 2} \sin \theta_{2}+m_{3} l_{1} l_{2} \sin \theta_{2}\right) \dot{\theta}_{2}-m_{3} l_{2} l_{\mathrm{c} 3} \sin \theta_{3} \dot{\theta}_{3}-m_{3} l_{1} l_{\mathrm{c} 3} \sin \left(\theta_{2}+\theta_{3}\right)\left(\dot{\theta}_{2}+\dot{\theta}_{3}\right) \\
V_{m 21}=\left(m_{2} l_{1} l_{\mathrm{c} 2} \sin \theta_{2}+m_{3} l_{1} l_{2} \sin \theta_{2}+m_{3} l_{1} l_{\mathrm{c} 3} \sin \left(\theta_{2}+\theta_{3}\right)\right) \dot{\theta}_{1}-m_{3} l_{2} l_{\mathrm{c} 3} \sin \theta_{3} \dot{\theta}_{3} \\
V_{m 31}=m_{3} l_{2} l_{\mathrm{c} 3} \sin \theta_{3}\left(\dot{\theta}_{1}+\dot{\theta}_{2}\right)+m_{3} l_{1} l_{\mathrm{c} 3} \sin \left(\theta_{2}+\theta_{3}\right) \dot{\theta}_{1} \\
V_{m 12}=-\left(m_{2} l_{1} l_{\mathrm{c} 2} \sin \theta_{2}+m_{3} l_{1} l_{2} \sin \theta_{2}\right)\left(\dot{\theta}_{1}+\dot{\theta}_{2}\right)-m_{3} l_{2} l_{\mathrm{c} 3} \sin \theta_{3} \dot{\theta}_{3}-m_{3} l_{1} l_{\mathrm{c} 3} \sin \left(\theta_{2}+\theta_{3}\right)\left(\dot{\theta}_{1}+\dot{\theta}_{2}+\dot{\theta}_{3}\right)
\end{gathered}
$$




$$
\begin{gathered}
V_{m 22}=-m_{3} l_{2} l_{\mathrm{c} 3} \sin \theta_{3} \dot{\theta}_{3} \\
V_{m 32}=m_{3} l_{2} l_{\mathrm{c} 3} \sin \theta_{3}\left(\dot{\theta}_{1}+\dot{\theta}_{2}\right) \\
V_{m 13}=-\left(m_{3} l_{2} l_{\mathrm{c} 3} \sin \theta_{3}+m_{3} l_{1} l_{\mathrm{c} 3} \sin \left(\theta_{2}+\theta_{3}\right)\right)\left(\dot{\theta}_{1}+\dot{\theta}_{2}+\dot{\theta}_{3}\right) \\
V_{m 23}=-m_{3} l_{2} l_{\mathrm{c} 3} \sin \theta_{3}\left(\dot{\theta}_{1}+\dot{\theta}_{2}+\dot{\theta}_{3}\right) \\
V_{m 33}=0
\end{gathered}
$$

Defining the following relationships:

$$
\begin{gathered}
c_{a}=m_{2} l_{1} l_{\mathrm{c} 2} \sin \theta_{2} \\
c_{b}=m_{3} l_{1} l_{2} \sin \theta_{2} \\
c_{c}=m_{3} l_{2} l_{\mathrm{c} 3} \sin \theta_{3} \\
c_{d}=m_{3} l_{1} l_{\mathrm{c} 3} \sin \left(\theta_{2}+\theta_{3}\right)
\end{gathered}
$$

Replacing expressions (91) to (94) into equations (82) to (89), we arrive to:

$$
\begin{gathered}
V_{m 11}=-\left(c_{a}+c_{b}\right) \dot{\theta}_{2}-c_{c} \dot{\theta}_{3}-c_{d}\left(\dot{\theta}_{2}+\dot{\theta}_{3}\right) \\
V_{m 21}=\left(c_{a}+c_{b}+c_{d}\right) \dot{\theta}_{1}-c_{c} \dot{\theta}_{3} \\
V_{m 31}=c_{c}\left(\dot{\theta}_{1}+\dot{\theta}_{2}\right)+c_{d} \dot{\theta}_{1} \\
V_{m 12}=-\left(c_{a}+c_{b}\right)\left(\dot{\theta}_{1}+\dot{\theta}_{2}\right)-c_{c} \dot{\theta}_{3}-c_{d}\left(\dot{\theta}_{1}+\dot{\theta}_{2}+\dot{\theta}_{3}\right) \\
V_{m 22}=-c_{c} \dot{\theta}_{3} \\
V_{m 32}=c_{c}\left(\dot{\theta}_{1}+\dot{\theta}_{2}\right) \\
V_{m 13}=-\left(c_{c}+c_{d}\right)\left(\dot{\theta}_{1}+\dot{\theta}_{2}+\dot{\theta}_{3}\right) \\
V_{m 23}=-c_{c}\left(\dot{\theta}_{1}+\dot{\theta}_{2}+\dot{\theta}_{3}\right) \\
V_{m 33}=0
\end{gathered}
$$

\subsection{Appendix D: Regression matrix for 3 DOF}

Defining relationships (104) and (105), and considering the relationships defined by means of equations (65) to (68), we have: 


$$
\begin{aligned}
& Q_{3}=\dot{q}_{d 3}+\lambda_{2} e_{3} \\
& \dot{Q}_{3}=\ddot{q}_{d 3}+\lambda_{3} \dot{e}_{3} \\
& Y_{11}=\frac{M_{11} \dot{Q}_{1}+M_{12} \dot{Q}_{2}+M_{13} \dot{Q}_{3}}{m_{1}} /_{\substack{m_{2}=0 \\
m_{3}=0}}+\frac{V_{m 11} Q_{1}+V_{m 12} Q_{2}+V_{m 13} Q_{3}}{m_{1}} /_{\substack{m_{2}=0 \\
m_{3}=0}}+\frac{G_{1}}{m_{1}} \int_{\substack{m_{2}=0 \\
m_{3}=0}} \\
& Y_{12}=\frac{M_{11} \dot{Q}_{1}+M_{12} \dot{Q}_{2}+M_{13} \dot{Q}_{3}}{m_{2}} /_{\substack{m_{1}=0 \\
m_{3}=0}}+\frac{V_{m 11} Q_{1}+V_{m 12} Q_{2}+V_{m 13} Q_{3}}{m_{2}} /_{\substack{m_{1}=0 \\
m_{3}=0}}+\left.\frac{G_{1}}{m_{2}}\right|_{\substack{m_{1}=0 \\
m_{3}=0}} \\
& Y_{13}=\frac{M_{11} \dot{Q}_{1}+M_{12} \dot{Q}_{2}+M_{13} \dot{Q}_{3}}{m_{3}} /_{\substack{m_{1}=0 \\
m_{2}=0}}+\frac{V_{m 11} Q_{1}+V_{m 12} Q_{2}+V_{m 13} Q_{3}}{m_{3}} / \overbrace{\substack{m_{1}=0 \\
m_{2}=0}}+\frac{G_{1}}{m_{3}} /_{\substack{m_{1}=0 \\
m_{2}=0}} \\
& Y_{21}=\frac{M_{21} \dot{Q}_{1}+M_{22} \dot{Q}_{2}+M_{23} \dot{Q}_{3}}{m_{1}} /_{\substack{m_{2}=0 \\
m_{3}=0}}+\frac{V_{m 21} Q_{1}+V_{m 22} Q_{2}+V_{m 23} Q_{3}}{m_{1}} / \int_{\substack{m_{2}=0 \\
m_{3}=0}}+\frac{G_{2}}{m_{1}} \int_{\substack{m_{2}=0 \\
m_{3}=0}} \\
& Y_{22}=\frac{M_{21} \dot{Q}_{1}+M_{22} \dot{Q}_{2}+M_{23} \dot{Q}_{3}}{m_{2}} /_{\substack{m_{1}=0 \\
m_{3}=0}}+\frac{V_{m 21} Q_{1}+V_{m 22} Q_{2}+V_{m 23} Q_{3}}{m_{2}} /_{\substack{m_{1}=0 \\
m_{3}=0}}+\frac{G_{2}}{m_{2}} /_{\substack{m_{1}=0 \\
m_{3}=0}} \\
& Y_{23}=\frac{M_{21} \dot{Q}_{1}+M_{22} \dot{Q}_{2}+M_{23} \dot{Q}_{3}}{m_{3}} \int_{\substack{m_{1}=0 \\
m_{2}=0}}+\frac{V_{m 21} Q_{1}+V_{m 22} Q_{2}+V_{m 23} Q_{3}}{m_{3}} / \int_{\substack{m_{1}=0 \\
m_{2}=0}}+\frac{G_{2}}{m_{3}} \int_{\substack{m_{1}=0 \\
m_{2}=0}} \\
& Y_{31}=\frac{M_{31} \dot{Q}_{1}+M_{32} \dot{Q}_{2}+M_{33} \dot{Q}_{3}}{m_{1}} /_{\substack{m_{2}=0 \\
m_{3}=0}}+\frac{V_{m 31} Q_{1}+V_{m 32} Q_{2}+V_{m 33} Q_{3}}{m_{1}} /_{\substack{m_{2}=0 \\
m_{3}=0}}+\frac{G_{3}}{m_{1}} /_{\substack{m_{2}=0 \\
m_{3}=0}} \\
& Y_{32}=\frac{M_{31} \dot{Q}_{1}+M_{32} \dot{Q}_{2}+M_{33} \dot{Q}_{3}}{m_{2}} /_{\substack{m_{1}=0 \\
m_{3}=0}}+\frac{V_{m 31} Q_{1}+V_{m 32} Q_{2}+V_{m 33} Q_{3}}{m_{2}} / \overbrace{\substack{m_{1}=0 \\
m_{3}=0}}+\frac{G_{3}}{m_{2}} /_{\substack{m_{1}=0 \\
m_{3}=0}} \\
& Y_{33}=\frac{M_{31} \dot{Q}_{1}+M_{32} \dot{Q}_{2}+M_{33} \dot{Q}_{3}}{m_{3}} /_{\substack{m_{1}=0 \\
m_{2}=0}}+\left.\frac{V_{m 31} Q_{1}+V_{m 32} Q_{2}+V_{m 33} Q_{3}}{m_{3}}\right|_{\substack{m_{1}=0 \\
m_{2}=0}}+\frac{G_{3}}{m_{3}} /_{\substack{m_{1}=0 \\
m_{2}=0}} \\
& Y_{11}=\left(I_{1 \mathrm{zz}} / m_{1}+l_{\mathrm{c} 1}^{2}\right) \dot{Q}_{1}
\end{aligned}
$$

$Y_{12}=-l_{1} l_{\mathrm{c} 2} \sin \theta_{2} \dot{\theta}_{2} Q_{1}-l_{1} l_{\mathrm{c} 2} \sin \theta_{2}\left(\dot{\theta}_{1}+\dot{\theta}_{2}\right) Q_{2}+\left(I_{2 \mathrm{zz}} / m_{2}+l_{1}^{2}+l_{\mathrm{c} 2}^{2}+2 l_{1} l_{\mathrm{c} 2} \cos \theta_{2}\right) \dot{Q}_{1}+$

$$
\left(I_{2 \mathrm{zz}} / m_{2}+l_{\mathrm{c} 2}^{2}+l_{1} l_{\mathrm{c} 2} \cos \theta_{2}\right) \dot{Q}_{2}
$$$$
Y_{13}=-\left(\left(l_{1} l_{2} \sin \theta_{2}+l_{1} l_{\mathrm{c} 3} \sin \left(\theta_{2}+\theta_{3}\right)\right) \dot{\theta}_{2}+\left(l_{2} l_{\mathrm{c} 3} \sin \theta_{3}+l_{1} l_{\mathrm{c} 3} \sin \left(\theta_{2}+\theta_{3}\right)\right) \dot{\theta}_{3}\right) Q_{1}+
$$$$
-\left(\left(l_{1} l_{2} \sin \theta_{2}+l_{1} l_{\mathrm{c} 3} \sin \left(\theta_{2}+\theta_{3}\right)\right)\left(\dot{\theta}_{1}+\dot{\theta}_{2}\right)\right) Q_{2}+\left(l_{2} l_{\mathrm{c} 3} \sin \theta_{3}+l_{1} l_{\mathrm{c} 3} \sin \left(\theta_{2}+\theta_{3}\right)\right)
$$$$
\dot{\theta}_{3} Q_{2}-\left(l_{2} l_{\mathrm{c} 3} \sin \theta_{3}+l_{1} l_{\mathrm{c} 3} \sin \left(\theta_{2}+\theta_{3}\right)\right)\left(\dot{\theta}_{1}+\dot{\theta}_{2}+\dot{\theta}_{3}\right) Q_{3}+\left(I_{3 \mathrm{zz}} / m_{3}+l_{1}^{2}+l_{2}^{2}+l_{\mathrm{c} 3}^{2}\right.
$$

$\left.\left.+2 l_{1} l_{2} \cos \theta_{2}+l_{2} l_{\mathrm{c} 3} \cos \theta_{3}+l_{1} l_{\mathrm{c} 3} \cos \left(\theta_{2}+\theta_{3}\right)\right)\right) \dot{Q}_{1}+\left(I_{3 \mathrm{zz}} / m_{3}+l_{2}^{2}+l_{\mathrm{c} 3}^{2}+l_{1} l_{2} \cos \theta_{2}\right.$

$\left.+2 l_{2} l_{\mathrm{c} 3} \cos \theta_{3}+l_{1} l_{\mathrm{c} 3} \cos \left(\theta_{2}+\theta_{3}\right)\right) \dot{Q}_{2}+\left(l_{\mathrm{c} 3}^{2}+l_{2} l_{\mathrm{c} 3} \cos \theta_{3}+l_{1} l_{\mathrm{c} 3} \cos \left(\theta_{2}+\theta_{3}\right)\right) \dot{Q}_{3}$ 


$$
\begin{gathered}
Y_{21}=0 \\
Y_{22}=l_{1} l_{\mathrm{c} 2} \sin \theta_{2} \dot{\theta}_{1} Q_{1}+\left(I_{2 \mathrm{zz}} / m_{2}+\left(l_{\mathrm{c} 2}^{2}+l_{1} l_{\mathrm{c} 2} \cos \theta_{2}\right)\right) \dot{Q}_{1}+\left(I_{2 \mathrm{zz}} / m_{2}+l_{\mathrm{c} 2}^{2}\right) \dot{Q}_{2} \\
Y_{23}=\left(l_{1} l_{2} \sin \theta_{2} \dot{\theta}_{1}+l_{1} l_{\mathrm{c} 3} \sin \left(\theta_{2}+\theta_{3}\right) \dot{\theta}_{1}-l_{2} l_{\mathrm{c} 3} \sin \theta_{3} \dot{\theta}_{3}\right) Q_{1}-l_{2} l_{\mathrm{c} 3} \sin \theta_{3} \dot{\theta}_{3} Q_{2}-l_{2} l_{\mathrm{c} 3} \sin \theta_{3} \\
\left(\dot{\theta}_{1}+\dot{\theta}_{2}+\dot{\theta}_{3}\right) Q_{3}+\left(I_{3 \mathrm{zz}} / m_{3}+l_{2}^{2}+l_{\mathrm{c} 3}^{2}+l_{1} l_{2} \cos \theta_{2}+2 l_{2} l_{\mathrm{c} 3} \cos \theta_{3}+l_{1} l_{\mathrm{c} 3} \cos \left(\theta_{2}+\theta_{3}\right)\right) \\
\dot{Q}_{1}+\left(I_{3 \mathrm{zz}} / m_{3}+l_{2}^{2}+l_{\mathrm{c} 3}^{2}+2 l_{2} l_{\mathrm{c} 3} \cos \theta_{3}\right) \dot{Q}_{2}+\left(I_{3 \mathrm{zz}} / m_{3}+l_{\mathrm{c} 3}^{2}+l_{2} l_{\mathrm{c} 3} \cos \theta_{3}\right) \dot{Q}_{3} \\
Y_{31}=0 \\
Y_{32}=0 \\
Y_{33}=\left(l_{2} l_{\mathrm{c} 3} \sin \theta_{3}\left(\dot{\theta}_{1}+\dot{\theta}_{2}\right)+l_{1} l_{\mathrm{c} 3} \sin \left(\theta_{2}+\theta_{3}\right) \dot{\theta}_{1}\right) Q_{1}+l_{2} l_{\mathrm{c} 3} \sin \theta_{3}\left(\dot{\theta}_{1}+\dot{\theta}_{2}\right) Q_{2}+ \\
\left(I_{3 \mathrm{zz}} / m_{3}+l_{\mathrm{c} 3}^{2}+l_{2} l_{\mathrm{c} 3} \cos \theta_{3}+l_{1} l_{\mathrm{c} 3} \cos \left(\theta_{2}+\theta_{3}\right)\right) \dot{Q}_{1}+\left(I_{3 \mathrm{zz}} / m_{3}+l_{\mathrm{c} 3}^{2}+l_{2} l_{\mathrm{c} 3}\right. \\
\left.\cos \theta_{3}\right) \dot{Q}_{2}+\left(I_{3 \mathrm{zz}} / m_{3}+l_{\mathrm{c} 3}^{2}\right) \dot{Q}_{3}
\end{gathered}
$$

Defining relationships (124) to (129), and considering the relationships defined in (78) and (79), we have:

$$
\begin{gathered}
y_{b}=l_{1} l_{2} \sin \theta_{2} \\
y_{c}=l_{2} l_{\mathrm{c} 3} \sin \theta_{3} \\
y_{d}=l_{1} l_{\mathrm{c} 3} \sin \left(\theta_{2}+\theta_{3}\right) \\
y_{B}=l_{1} l_{2} \cos \theta_{2} \\
y_{C}=l_{2} l_{\mathrm{c} 3} \cos \theta_{3} \\
y_{D}=l_{1} l_{\mathrm{c} 3} \cos \left(\theta_{2}+\theta_{3}\right)
\end{gathered}
$$




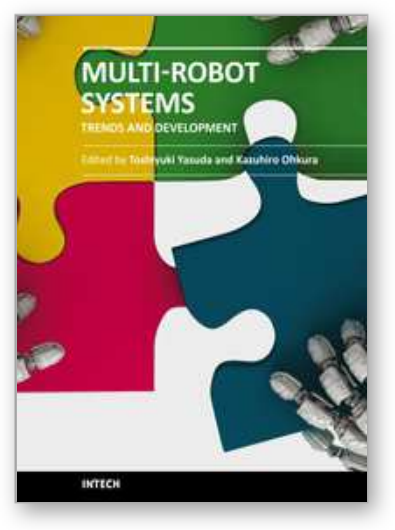

\author{
Multi-Robot Systems, Trends and Development \\ Edited by Dr Toshiyuki Yasuda
}

ISBN 978-953-307-425-2

Hard cover, 586 pages

Publisher InTech

Published online 30, January, 2011

Published in print edition January, 2011

This book is a collection of 29 excellent works and comprised of three sections: task oriented approach, bio inspired approach, and modeling/design. In the first section, applications on formation, localization/mapping, and planning are introduced. The second section is on behavior-based approach by means of artificial intelligence techniques. The last section includes research articles on development of architectures and control systems.

\title{
How to reference
}

In order to correctly reference this scholarly work, feel free to copy and paste the following:

Claudio Urrea Oñate and John Kern (2011). Modeling, Simulation and Control of 3-DOF Redundant Fault Tolerant Robots by Means of Adaptive Inertia, Multi-Robot Systems, Trends and Development, Dr Toshiyuki Yasuda (Ed.), ISBN: 978-953-307-425-2, InTech, Available from: http://www.intechopen.com/books/multirobot-systems-trends-and-development/modeling-simulation-and-control-of-3-dof-redundant-fault-tolerantrobots-by-means-of-adaptive-inerti

\section{INTECH}

open science | open minds

\section{InTech Europe}

University Campus STeP Ri

Slavka Krautzeka 83/A

51000 Rijeka, Croatia

Phone: +385 (51) 770447

Fax: +385 (51) 686166

www.intechopen.com

\section{InTech China}

Unit 405, Office Block, Hotel Equatorial Shanghai

No.65, Yan An Road (West), Shanghai, 200040, China

中国上海市延安西路65号上海国际贵都大饭店办公楼 405 单元

Phone: +86-21-62489820

Fax: +86-21-62489821 
(C) 2011 The Author(s). Licensee IntechOpen. This chapter is distributed under the terms of the Creative Commons Attribution-NonCommercialShareAlike-3.0 License, which permits use, distribution and reproduction for non-commercial purposes, provided the original is properly cited and derivative works building on this content are distributed under the same license. 